Case Report

\title{
Partial and full own-body illusions of epileptic origin in a child with right temporoparietal epilepsy
}

\author{
Lukas Heydrich $^{\mathrm{a}, \mathrm{b}}$, Christophe Lopez ${ }^{\mathrm{a}, \mathrm{c}}$, Margitta Seeck ${ }^{\mathrm{b}}$, Olaf Blanke ${ }^{\mathrm{a}, \mathrm{b}, *}$ \\ a Laboratory of Cognitive Neuroscience, Brain Mind Institute, Ecole Polytechnique Fédérale de Lausanne, Lausanne, Switzerland \\ b Department of Neurology, University Hospital, Geneva, Switzerland \\ c Institut für Psychologie, Abteilung für Kognitive Psychologie, Wahrnehmung und Methodenlehre, University of Bern, Bern, Switzerland
}

\section{A R T I C L E I N F O}

\section{Article history:}

Received 9 December 2010

Revised 4 January 2011

Accepted 5 January 2011

Available online 21 February 2011

\section{Keywords:}

Epilepsy

Pediatric neurology

Behavioral neurology

Out-of-body experience

Somatoparaphrenia

\begin{abstract}
A B S T R A C T
Partial and full own-body illusions of neurological origin have been claimed crucial to understand the contribution of bodily experience and perception to self-consciousness. Whereas partial body illusions are relatively common and well defined, much less is known about full own-body illusions, and even less is known about these illusions in children. Here we describe a 10-year-old patient with the association of partial and full own-body illusions (somatoparaphrenia and out-of-body experience) that occurred sequentially during an epileptic seizure caused by right temporoparietal epilepsy. This report shows that partial and full own-body illusions share functional and neuroanatomical properties and highlights the importance of the right temporoparietal junction for bodily self-consciousness. This is the first report of out-of-body experiences in a child with focal epilepsy.
\end{abstract}

(c) 2011 Elsevier Inc. All rights reserved.

\section{Introduction}

The careful study of neurological patients with abnormal bodily experiences [1] has been suggested to further the understanding of the multisensory and sensorimotor brain mechanisms underlying bodily self-consciousness. Thus, partial own-body illusions, like the transformation [2], disconnection [3], and disownership (e.g., somatoparaphrenia) [4] of a part of one's body, are thought to be of relevance and have been linked to damage to the right posterior parietal cortex [3] and right posterior insula [5].

Full own-body illusions, including out-of-body experiences (OBEs), are less often studied. During OBEs, patients have the sensation of being localized outside their physical body and of seeing themselves and the environment from an elevated first-person perspective [1]. OBEs have been reported in most instances after damage to, or interference with, the right temporoparietal junction or right posterior parietal cortex, but have also been observed in nonlesional epilepsy, after cardiogenic syncope, and in up to about $8-15 \%$ of the healthy population $[1,6-9]$. OBEs affect the body as a whole, and it has recently been proposed that they are particularly relevant for the experimental and neurological study of self-consciousness [10]. However, despite the seemingly close neuroanatomical overlap and the proposed association of partial and full

\footnotetext{
* Corresponding author at: Laboratory of Cognitive Neuroscience, Brain Mind Institute, Ecole Polytechnique Fédérale de Lausanne (EPFL), Swiss Federal Institute of Technology, Station 19, 1015 Lausanne, Switzerland. Fax: + 41216939625.

E-mail address: olaf.blanke@epfl.ch (O. Blanke).
}

own-body illusions [1,2], patients experiencing both illusions have rarely been reported. Moreover, to date, full own-body illusions, such as OBEs caused by focal brain damage, and their association with other bodily illusions have not been reported in children.

Here we describe the rare case of a 10 -year-old patient who experienced ictal somatoparaphrenia followed by an OBE during a complex partial seizure originating in the right temporoparietal lobe. We discuss the association of partial and full own-body illusions and the evidence for overlapping and distinct underlying mechanisms in right temporoparietal cortex, and highlight the semiological importance of such phenomena in adults and in children.

\section{Report of the case}

The patient is a 10-year-old, right-handed boy who was hospitalized for epilepsy evaluation. Although he had his first generalized seizure 2 days after birth, his early development was normal. Under phenobarbital treatment there were no epileptic seizures until the age of 2 years, when he started to have recurrent complex partial seizures with secondary generalization (frequency: one per month). Seizures were characterized by stereotypical clonic movements of the left upper extremity (rarely of left lower extremity) and were usually preceded by somatosensory sensations (paresthesias) affecting the left hand and cheek. Clonic movements of the left arm were usually followed by a head-eye deviation to the left, left hemiparesis, and secondary generalization with loss of consciousness.

We recorded one ictal episode during his hospitalization. The patient announced the habitual aura (paresthesia of the left hand), 
still able to speak and with intact naming. He then elevated and pointed to his left hand, indicating verbally that it hurt. This was followed ( 4 minutes) by clonic movements of the left arm, and the patient repeatedly asking his mother whether the left hand was his own hand ("Is this my hand?") (ictal somatoparaphrenia). Later, he fearfully exclaimed that another person was moving the left arm ("Look, the arm is moving. Who is moving the arm?") (2 minutes). This was followed by a tonic deviation of the head to the left, loss of consciousness, and secondary tonic-clonic generalization. The entire seizure lasted 14 minutes. Later, the patient reported spontaneously that after his partial own-body illusions and before losing consciousness, he had experienced a sensation of lightness and floating, followed by a vivid sensation that he was projected out of his physical body to an elevated position within the hospital room under the ceiling. He had the impression of seeing the room and his mother from this elevated visual perspective, but not his own body (no autoscopy). This was followed by a continued sensation of projection and the experience of a higher level of elevation as if he were flying above the hospital and eventually above the "world" ("It feels like I am flying way above the world but I know this can't be true. I feel light, very light!") (see Supplementary Fig. 1).
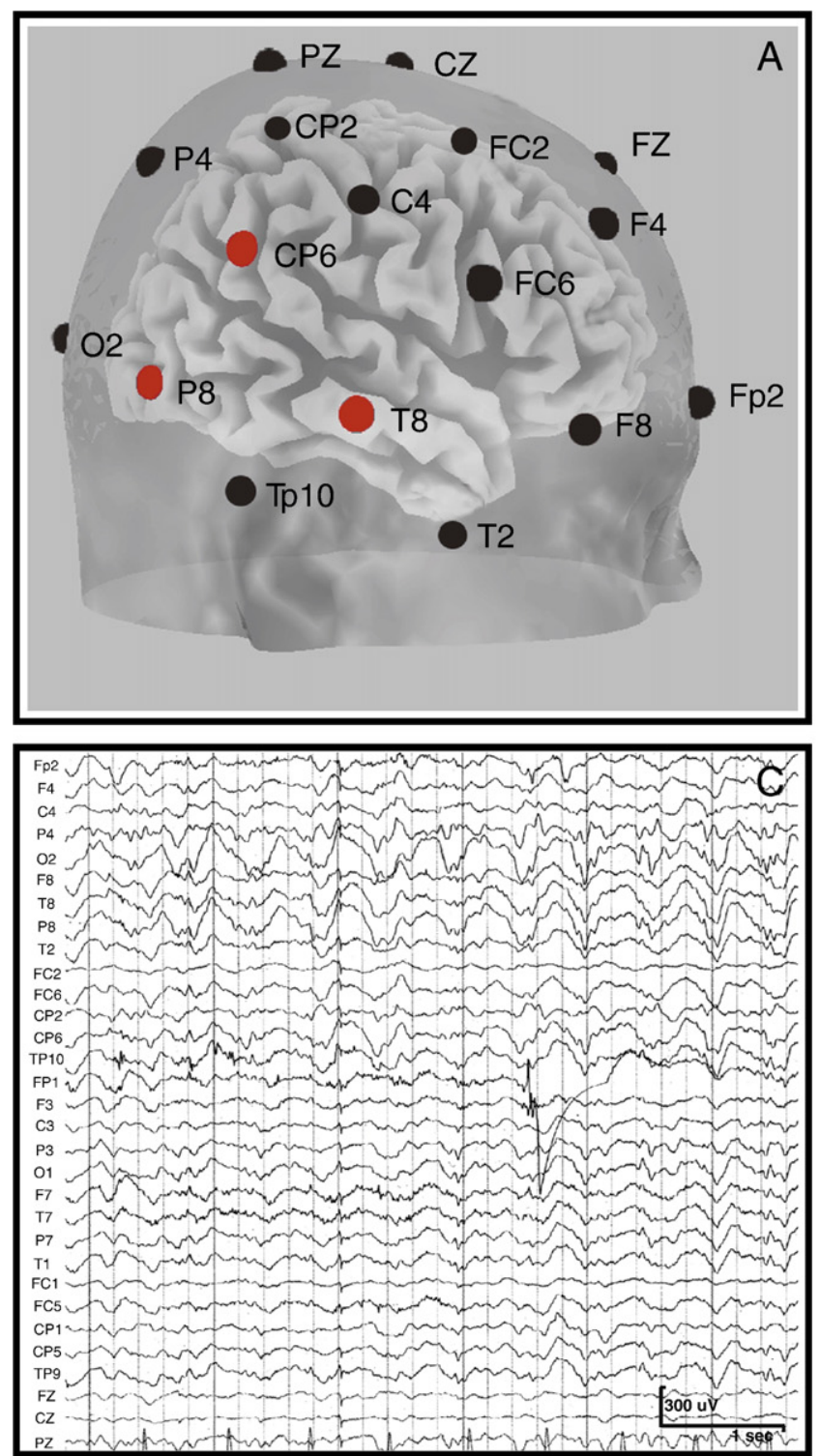

The ictal EEG revealed the seizure onset in the right parietotemporal region (Fig. 1B, electrodes T8, P8, CP6) with prolonged, 4-minute-long, focal rapid rhythms and slow waves. Concomitant with clonic movements of the left arm, slow waves and rapid rhythms spread to electrodes $\mathrm{P} 4$ and $\mathrm{O2}$, and were followed by sharp waves over right hemispheric posterior regions (Fig. 1C) and bilateral propagation after 14 minutes (Fig. 1D). No epileptic activity was recorded over anterior temporal regions (electrodes T2, F8) during the whole episode. Interictal EEG recordings suggested a right posterior temporal focus.

Magnetic resonance imaging revealed an ischemic lesion in the right angular gyrus (Fig. 2A), most probably a result of perinatal asphyxia, as well as right mesiotemporal sclerosis. Interictal positron emission tomography showed hypometabolism in the right angular gyrus, the right inferior parietal lobe, and the right anterior mesiotemporal lobe (Fig. 2B).

\section{Discussion}

We describe a rare case of an OBE of focal epileptic origin, reported by a young child, which was associated with contralesional paroxysmal partial own body illusions (ictal somatoparaphrenia). MRI and PET
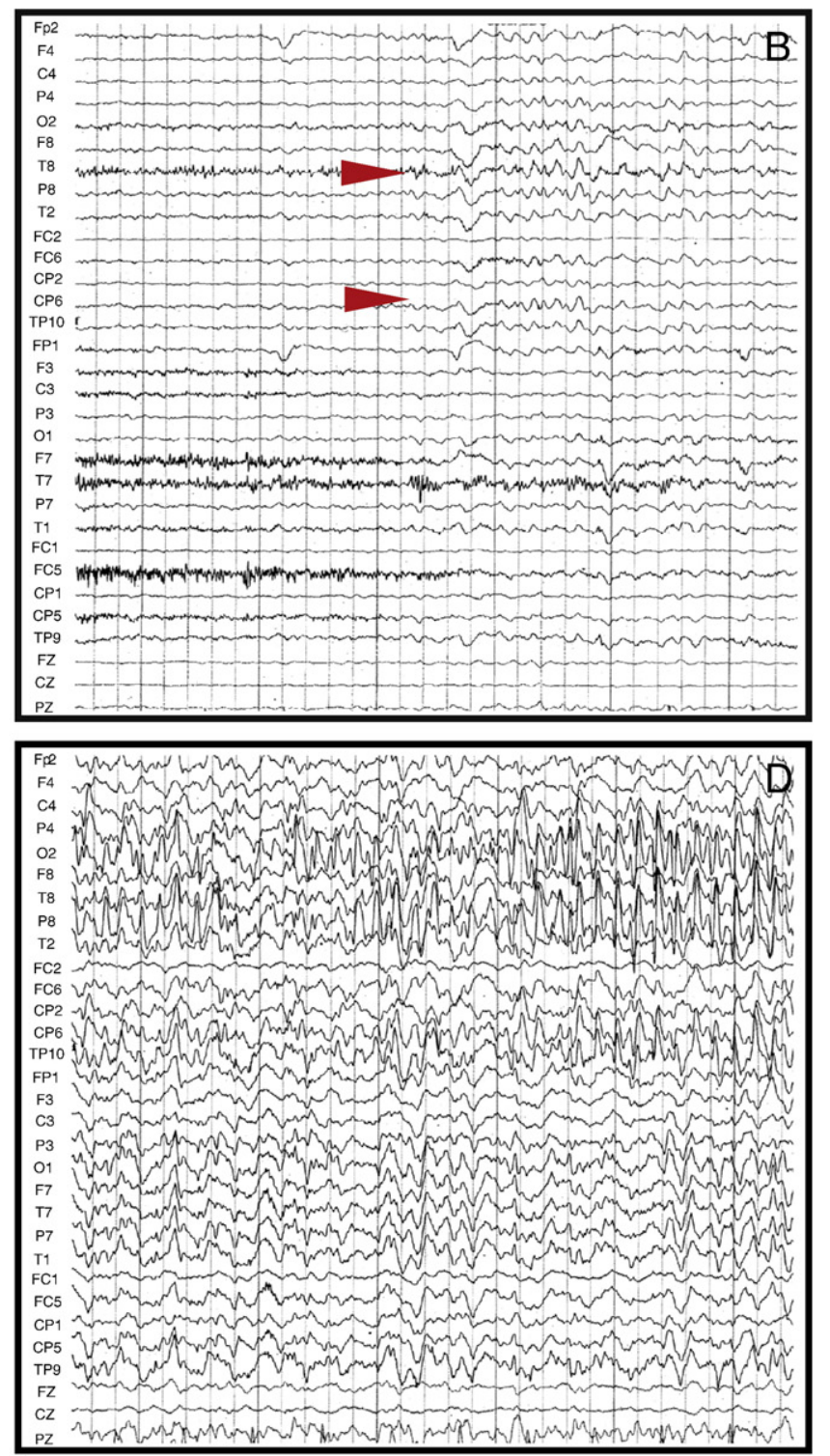

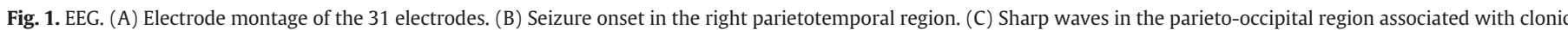
movements. (D) Bilateral propagation and secondary generalization associated with out-of-body experience. 

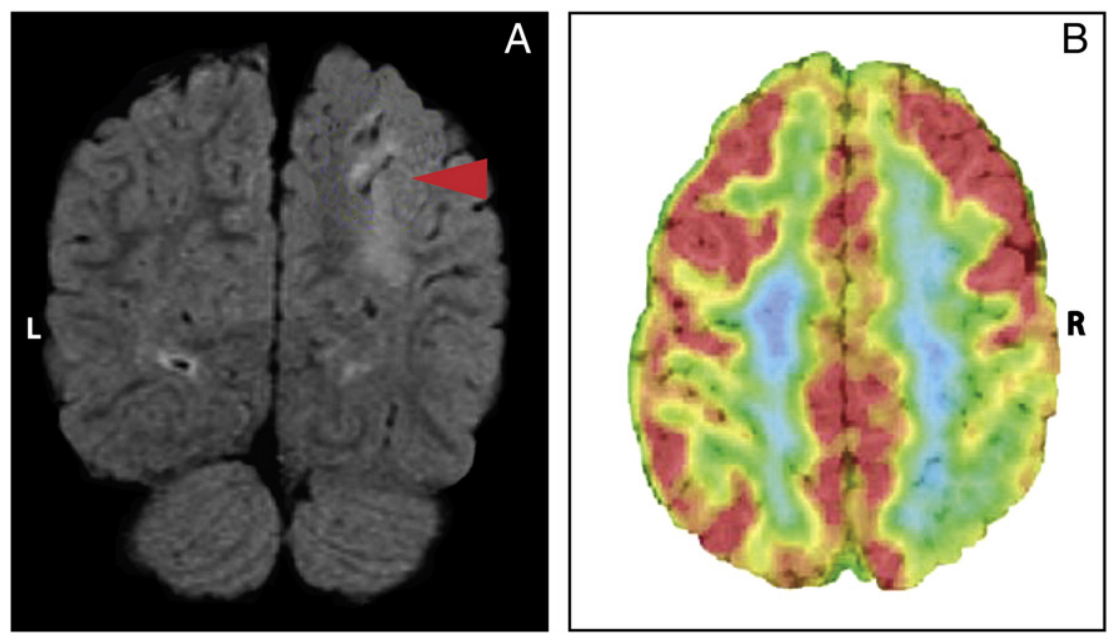

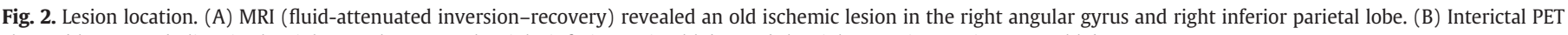
showed hypometabolism in the right angular gyrus, the right inferior parietal lobe, and the right anterior mesiotemporal lobe.

revealed an ischemic lesion affecting the right angular gyrus and right mesiotemporal sclerosis. Ictal EEG, however, demonstrated right posterior parietal and lateral temporal activity, which subsequently spread to the right posterior parietal lobe and the left hemisphere.

The somatosensory ictal semiology and the later association of somatoparaphrenia with an OBE are compatible with an origin of the seizure in parietotemporal and/or posterior parietal cortex. Although there is a gross neuroanatomical association in posterior temporal and posterior parietal cortex in patients with partial and full own-body illusions, the association has rarely been reported in the same patient. Thus, the present case allows us to dissociate both phenomena temporally and anatomically because of their successive ictal appearance, suggesting different brain mechanisms [1].

Somatoparaphrenia has been associated with damage to posterior parietal cortex, posterior superior temporal cortex [4] and right posterior insula [5]. OBEs have been associated with the right angular, supramarginal, and superior temporal gyrus [1], but also with right frontotemporal lesions $[9,11]$, concordant with the right hemisphere implication in the present patient. A possible association between partial and full own-body illusions was proposed by Devinsky et al. [9], who associated both phenomena with posterior temporal cortex, further supported by the demonstration that electrical stimulation at the right temporoparietal junction leads to partial as well as full ownbody illusions [1].

We argue that the sequential ictal bodily experiences of paresthesia, somatoparaphrenia, and an OBE in our patient suggest that ictal discharges at seizure onset were centered in anterior parietal regions (e.g., hand area in the postcentral gyrus and adjacent parts of the intraparietal sulcus), with subsequent spread to posterior parietal cortex, posterior insula, and related vestibular cortex [12]. This is also compatible with the ictal EEG findings and PET and MRI. Our patient's sequence of somatosensory hand sensations (paresthesia) to multisensory hand sensations (somatoparaphrenia) to multisensory full body sensations (OBE) may thus be compatible with propagation from unimodal somatosensory regions to multimodal somatosensory regions representing body parts and then the full body. The feeling that left hand movements were caused by another individual resembles the so-called "alien hand syndrome" during which movements of a body part are perceived as generated by another or alien person. This has been reported after damage to the posterior parietal cortex [13] and electrical cortical stimulation of the postcentral gyrus [14], which is anatomically compatible with the present case.

To the best of our knowledge, OBEs caused by focal brain damage have not been described in children. Although OBEs have been described in healthy children, the reported incidence is surprisingly low (2\%) as compared with that in adults [7]. There are several anecdotal reports of OBEs in children during high typhoid fever [15] and severe illness [16]. However, the present case is the first showing OBE caused by focal brain damage and partial epilepsy in a child. It supports the importance of addressing complex own-body illusions not only in adults, but also in children during the evaluation of epilepsy and other neurological conditions. The present case suggests that the rarity of OBEs and somatoparaphrenia of neurological origin may not be related to their general absence, but rather to underreporting of symptoms that are rarely spontaneously mentioned by the patient and rarely asked for by the examiner. Thus, it seems important to explicitly ask for this potentially more frequent symptom in patients with epilepsy and other neurological disorders [17].

In conclusion, we argue that the prolonged ictal sequence of hand somatosensory sensations to somatoparaphrenic hand sensations to a full body illusion associated with vestibular sensations suggests a propagation of ictal discharges from anterior parietal cortex to posterior parietal cortex involving the right posterior insular cortex and the right temporoparietal junction. This propagation is compatible with the functional roles of these regions in unimodal somatosensory and multimodal processing for body parts and the full body, respectively, which have been linked to different aspects of self-consciousness and own body processing $[5,10]$.

Supplementary material associated with this article can be found, in the online version, at doi:10.1016/j.yebeh.2011.01.008.

\section{Ethical approval}

We confirm that we have read the Journal's position on issues involved in ethical publication and affirm that this report is consistent with those guidelines.

\section{Conflict of interest statement}

None of the authors has any conflict of interest to disclose.

\section{Acknowledgments}

Thanks to Dr. Nguyen from the Hospital of Geneva for his help analyzing the MRI data and to Nathan Evans for his helpful comments on an earlier draft of the article. L.H. and M.S. are supported by the Swiss National Science Foundation (Grants 33CM30-124089, 33CM30123115, 320030-122073, 323530-123718, 3CM30-124115). O.B. and 
C.L. are supported by the Swiss National Science Foundation (Sinergia Grant CRSII1-125135: Balancing Self and Body).

\section{References}

[1] Blanke O, Landis T, Spinelli L, Seeck M. Out-of-body experience and autoscopy of neurological origin. Brain 2004;127(Pt 2):243-58.

[2] Hécaen H, Ajuriaguerra J. Méconnaissances et hallucinations corporelles. Paris: Masson; 1952.

[3] Heydrich L, Dieguez S, Grunwald T, Seeck M, Blanke O. Illusory own body perceptions: case reports and relevance for bodily self-consciousness. Conscious Cogn 2010;19:702-10.

[4] Vallar G, Ronchi R. Somatoparaphrenia: a body delusion. A review of the neuropsychological literature. Exp Brain Res 2009;192:533-51.

[5] Baier B, Karnath HO. Tight link between our sense of limb ownership and selfawareness of actions. Stroke 2008;39:486-8.

[6] Maillard L, Vignal JP, Anxionnat R, TaillandierVespignani L. Semiologic value of ictal autoscopy. Epilepsia 2004;45:391-4.

[7] Blackmore SJ, Wooffitt RC. Out-of-body experiences in young children. J Soc Psychical Res 1990;56:155-8.
[8] Brandt C, Kramme C, Storm H, Pohlmann-Eden B. Out-of-body experience and auditory and visual hallucinations in a patient with cardiogenic syncope: crucial role of cardiac event recorder in establishing the diagnosis. Epilepsy Behav 2009;15: 254-5.

[9] Devinsky O, Feldmann E, Burrowes K, Bromfield E. Autoscopic phenomena with seizures. Arch Neurol 1989;46:1080-8.

[10] Blanke O, Metzinger T. Full-body illusions and minimal phenomenal selfhood. Trends Cogn Sci 2009;13:7-13.

[11] Lopez C, Heydrich L, Seeck M, Blanke O. Abnormal self-location and vestibular vertigo in a patient with right frontal lobe epilepsy. Epilepsy Behav 2010;17:289-92.

[12] Kahane P, Hoffmann D, Minotti L, Berthoz A. Reappraisal of the human vestibular cortex by cortical electrical stimulation study. Ann Neurol 2003;54:615-24.

[13] Leiguarda R, Starkstein S, Nogues M, Berthier M, Arbelaiz R. Paroxysmal alien hand syndrome. J Neurol Neurosurg Psychiatry 1993;56:788-92.

[14] Boesebeck F, Ebner A. Paroxysmal alien limb phenomena due to epileptic seizures and electrical cortical stimulation. Neurology 2004;63:1725-7.

[15] Menninger-Lerchenthal E. Das Truggebilde der eigenen Gestalt. Berlin: S. Karger; 1935

[16] Morse M, Castillo P, Venecia D, Milstein J, Tyler DC. Childhood near-death experiences. Am J Dis Child 1986;140:1110-4.

[17] Brandt C, Brechtelsbauer D, Bien CG, Reiners K. Out-of-body experience as possible seizure symptom in a patient with a right parietal lesion. Nervenarzt 2005;76(1259): 61-2. 